\title{
Latest features in GaBI Journal, 2013, Issue 3
}

\section{Professor Philip Walson, MD}

The GaBI Journal again contains manuscripts covering a wide range of pertinent topics. In an Editorial, Dr Gianluigi Casadei reviews Italy's final position paper on biosimilars including the new price and reimbursement position from the Italian Medicines Agency (Agenzia Italiana del Farmaco, AIFA). Dr Casadei questions how much of a 'step forward' as well as just how 'progressive' these are. He suggests these may be too conservative since substitution decisions are left to the prescribers who should preferentially prescribe generics only if there is a clear economic benefit' to the Italian healthcare system. Finally, the author discusses why pricing discounts are 'likely to represent a barrier to entry for biosimilars'.

In the first of three Commentaries, Professor Teun van Gelder comments on recommendations of the European Society for Organ Transplantation (ESOT) Advisory Committee on Generic Substitution recently published in Transplant International. The ESOT recommendations are based on concerns about lack of generic to generic comparisons, uncontrolled substitution, and patient confusion. The author and ESOT conclude that proving bioequivalence does not justify uncontrolled substitution of generic immunosuppressant drugs.

In the second Commentary, Professor Andrea Laslop from the Austrian Agency for Health and Food Safety discusses the new challenges and opportunities for the use of biosimilar monoclonal antibodies in Europe. She makes the case for requiring additional testing for monoclonals versus previously approved, simpler biologicals as well as the need for improvements in pharmacovigilance, tracking and documentation. She also suggests that even with improved testing it is 'prudent to avoid multiple switches'.

In the third and final Commentary, Dr Brian Godman discusses ongoing prescribing initiatives in the Republic of Srpska (Bosnia and Herzegovina) by reviewing a paper published by Markovic-Pekovic et al. on the effects of recent reforms in the Republic of Srpska that described demand-side measures aimed at prescribing, restrictions and incentives designed to increase the use of low priced generics. The author points out that outcomes dispute claims that small countries will not be able to decrease costs through use of generics.

In the first of three Original Research papers, Mueller et al. describe a method for the production of recombinant parathyroid hormone (rPTH) fragment 1-34 in the methylotrophic yeast Hansenula polymorpha. They describe why they believe this new method is robust, efficient, and potentially cost saving. It is worth mentioning that the GaBIJournal is very interested in publishing manuscripts describing such 'methods' as well as critiques and comments by our readers.

In the second Original Research paper, Curto et al. present their analysis of 24 'public tenders' for 191 lots of three offpatent biologicals were actually handled by some of the autonomous regional authorities in Italy. They found a (difficult to justify) lack of actual competition in the diverse processes used by the bidders (manufacturers) and buyers (regional authorities) to provide these three biosimilar products (somatropin, epoetin and filgrastim).

In the final Original Research paper, Chiu et al. propose that the statistical method of generalized pivotal quantities should replace traditional bioequivalence methods because 'standard methods for bioequivalence assessment of small-molecule drug products cannot be directly applied to assessing biosimilarity of biosimilar products owing to some fundamental differences between small-molecule generic and large-molecule biosimilar drug products'.

In the first of two Review Articles, Mica et al. suggest ways that healthcare providers should evaluate critical supply chain factors when making formulary decisions

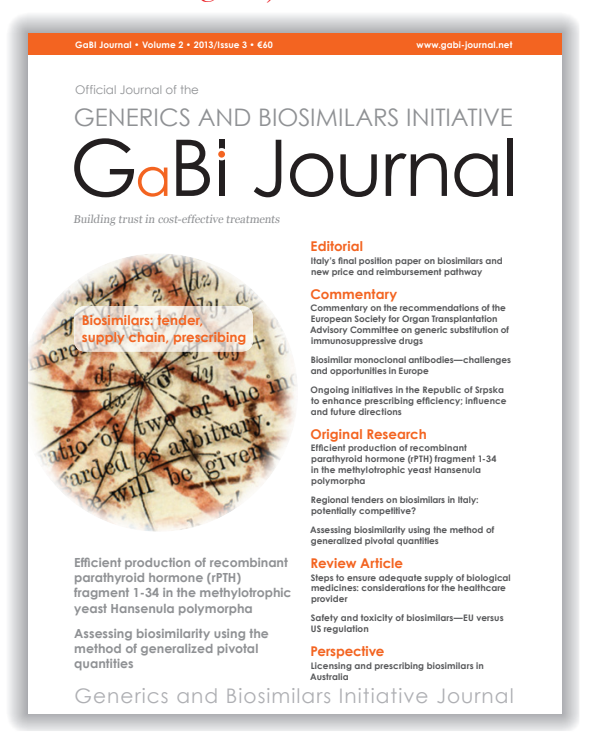

about biosimilar products. They also suggest that manufacturers and regulators should consider the value of making such information available and point out that this 'will become increasingly important as the number of marketed biologicals and the number of suppliers increase'.

In the other Review Article, Blank et al. review differences in how biosimilars are regulated in the EU and US and discuss why they believe harmonization is needed.

In the Perspective paper, Professor David Power describes how patient's requests to have biologicals delivered by community rather than hospital pharmacies 'has the potential to dilute cost savings and increase the complexity of pharmacovigilance'.

In the Regulatory paper, our GaBI Journal editor, Dr Michelle Derbyshire, summarizes legislation being considered in a number of US states that could limit biosimilars substitution.

The editors, editorial staff and I hope that our readers will find these manuscripts as stimulating and useful as we did and again encourage comments, critiques and manuscripts that either expand on these topics or discuss additional issues.

Professor Philip Walson, MD Editor-in-Chief, GaBiJournal

DOI: $10.5639 /$ gabij.2013.0203.032

Copyright (c) 2013 Pro Pharma Communications International 